\title{
La Spagna nello Zibaldone
}

\author{
Costanza Geddes da Filicaia \\ Università degli Studi di Macerata
}

\begin{abstract}
Il saggio in oggetto si propone di analizzare le carte zibaldoniche in cui Leopardi tratta della nazione spagnola, al fine di stabilire quale opinione esprima il poeta della condizione socio-antropologica, culturale, linguistico-letteraria della Spagna. Si osserva innanzitutto come, nell'ottica leopardiana, la realtà iberica versi in uno stato di decadenza rispetto a un glorioso passato. Tale opinione nasce probabilmente anche dal fatto che Giacomo ha una visione antiprogressiva della storia. A ciò si dovrà inoltre aggiungere che gli anni a cui risalgono i passi zibaldonici dedicati alla Spagna sono quelli del pessimismo storico, periodo in cui permane l'idea, del resto mai completamente abbandonata, di una perduta "età aurorale del mondo". Si nota poi che un interesse primario di Leopardi è quello di paragonare la Spagna con l'Italia, nell'evidente percezione di un'intrinseca affinità tra i due paesi. Si segnala inoltre che fra costumi italiani mutuati dalla Spagna vi sarebbe l'abitudine di attribuire titoli nobiliari lunghi e pomposi. In generale, la condizione italiana pare al poeta nettamente peggiore rispetto a quella spagnola, in quanto alcuni elementi di preservata naturalità consentono alla terra iberica di possedere maggior vitalità rispetto all'Italia. Si analizzano poi le carte zibaldoniche dedicate alle virtù guerriere degli spagnoli, alla descrizione delle loro tradizioni, dei costumi, della religiosità, soffermandosi inoltre su un curioso giudizio leopardiano di ordine letterario in base al quale nessuna opera spagnola avrebbe mai raggiunto fama internazionale.

Lultima parte del saggio è dedicata alle osservazioni di Giacomo sull'idioma spagnolo, dalle quali emerge l'opinione di una strettissima affinità tra l'Italia e la Spagna anche sul piano linguistico, tanto che molti lemmi sarebbero entrati nel lessico di una delle due lingue proveniendo dall'altra, senza essere in alcun modo percepiti come stranieri.
\end{abstract}

Parole chiave: Leopardi, Spagna, lingua spagnola, confronto fra Italia e Spagna.

\begin{abstract}
This paper analysese the Zibaldone letters, in which Leopardi makes observations on the Spanish nation, in an attempt to identify Leopardi's true opinion on the social, anthropological, cultural, linguistic and literary conditions of Spain. Above all, Leopardi believes that Spain lives in a condition of decline, especially in comparison with its glorious past. This opinion is probably born of Giacomo's anti-progressive view of history. In addition, the Zibaldone passages concerning Spain were written during the years of historic pessimism, in which it was widely believed that the «auroral era» had been lost from the world,
\end{abstract}


an idea which has not been completely abandoned. It emerges from this analysis that one of Leopardi's main interests is that to compare Spain and Italy, in an evident perception of an intimate affinity between the two countries. Among the customs that Italy derived from Spain, there is leaning towards awarding long, pompous titles of rank. On a whole, Leopardi believes that Italian condition is much worse than the Spanish one, because Spain has preserved some elements of natural spirit and therefore of vitality, while Italy lost it. In the Zibaldone letters there are also passages concerning Spanish virtue in war, the description of its customs and its religiousness. They also allude to a peculiar opinion of Leopardi that no Spanish literary work would ever attract international acclaim.

The last part of the essay concerns Leopardi's observations on Spanish language. He defends the idea of a very close linguistic affinity between Italian and Spanish. Many words have been included in the vocabulary of one of the two languages coming from the other language, without them being perceived as foreign words at all.

Key words: Leopardi, Spain, Spanish language, comparison of Italy and Spain.

Oggidì i viaggi più curiosi e più interessanti che si possono fare in Europa cioè nel paese incivilito, sono quelli ne' paesi meno inciviliti, cioè la Svizzera, la Spagna e simili, che tuttavia conservano qualche natura e proprietà. La descrizione de' costumi, de' caratteri, delle opinioni, delle usanze di questi paesi hanno sempre della varietà, della singolarità, della importanza, della curiosità. ${ }^{1}$

In questo passo zibaldonico, risalente al 5 marzo 1821, Giacomo Leopardi esprime in maniera sintetica ma chiara un'opinione sulla Spagna che avrà più volte modo di ribadire e di approfondire nello stesso Zibaldone: la nazione iberica, un tempo culla di grandi e raffinate civiltà, è divenuta barbara, arretrata, incapace di qualsiasi spinta propulsiva sia sul piano socio-politico che su quello letterario. ${ }^{2}$ Del resto, la Spagna è un paese dell'Europa meridionale: secondo Leopardi, se quei luoghi videro fiorire la cultura antica, nell'età moderna i centri culturali più vivaci appartengono invece all'area settentrionale del Continente, significativamente l'Inghilterra e la Germania. ${ }^{3}$ Tale mutamento sarebbe avvenuto in seguito a complesse vicende socio-politiche che hanno causato, tra le altre conseguenze, un generale «intorpidimento» dei costumi meridionali. ${ }^{4}$ Non si deve tuttavia trascurare il fatto che la visione leopardiana della

1. Giacomo LeOpard, Zibaldone di Pensieri, a cura di Giuseppe Pacella, Milano: Garzanti, 1991, p. 459.

2. Tale argomento è stato affrontato anche nel saggio di Clara FERRANTI, «Lo spagnolo nelle riflessioni linguistiche dello Zibaldone di Leopardi», in Rapporti culturali fra Italia e Spagna, VII Incontro, Macerata: Università degli Studi, 16-17 novembre 2000, supplemento a Quaderni di filologia e lingue romanze. Ricerche svolte nell'Università degli Studi di Macerata, terza serie, 15, 2000.

3. Si veda a questo proposito il saggio di Adriana MITESCU, «Lo "Zibaldone”, riflessioni sulle lingue straniere», in La corrispondenza imperfetta. Leopardi tradotto e traduttore, a cura di Anna Dolfi e Adriana Mitescu, Roma: Bulzoni, 1990, p. 41-57.

4. Si veda, a questo proposito, il saggio di Maria de las Nieves MuÑIz MuÑIZ, «La Spagna bifronte dello "Zibaldone" (ancora su Leopardi progressivo)", in Actes du Colloque Inter- 
storia è radicalmente antiprogressiva e tende quindi a rilevare più gli eventuali stati di decadenza di una nazione o di un popolo che gli elementi di miglioramento. ${ }^{5}$ Inoltre, l'opposizione antichi-moderni si risolve sempre, nella meditazione del poeta, a favore dei primi: se nella fase del così detto pessimismo storico (1823-1824) c'è da parte di Leopardi una minuziosa spiegazione dei motivi per cui gli antichi possono ritenersi superiori ai moderni (maggior vigore fisico, entusiasmo, culto della immaginazione, delle illusioni e della virtù), anche dopo la svolta in direzione del pessimismo cosmico resta radicata l'idea di una minore infelicità degli antichi o, più precisamente, di una minore consapevolezza della propria infelicità. ${ }^{6}$ L'immagine del mondo antico conserverà sempre, per Giacomo, un'aura favolosa ed edenica: anche in quest'ottica si deve dunque inquadrare il giudizio su una pristina grandezza spagnola, a cui sarebbe seguito uno stato irrimediabile di decadenza. Tale situazione accomunerebbe peraltro la Spagna e l'Italia, paesi simili non solo per costumi e usanze, ma anche per la loro condizione di decaduta nobiltà.

Prima di addentrarci nello specifico delle riflessioni zibaldoniche sulla Spagna, sarà opportuno precisare che Leopardi mostra, nello Zibaldone, una diffusa attenzione per le caratteristiche antropologiche, sociali, culturali e linguistiche di vari popoli. Sono oggetto del suo interesse, oltre agli Spagnoli e, ovviamente, agli Italiani, i Celti, i Cinesi, gli Ebrei, i Francesi, i Greci, gli Inglesi, i Persiani, i Polacchi, i Russi, gli Svedesi, gli Svizzeri, i Tedeschi. Molte volte, inoltre, il poeta si sofferma su vari aspetti della lingua sanscrita, largamente parlata nel sub-continente indiano. Non sorprenderà, dunque, il fatto che nei passi dedicati alla Spagna gli argomenti affrontati siano molteplici: da meditazioni di carattere storico-politico, a osservazioni su usi e costumi, a riflessioni letterarie e linguistiche. Va inoltre osservato che la stesura di quasi tutti questi brani risale al triennio 1820-1823: siamo quindi nella fase del pessimismo storico, durante la quale l'idea di una superiorità degli antichi rispetto ai moderni, ancorché destinata, come già si diceva, a permanere successivamente, è tuttavia sostenuta in maniera particolarmente forte e decisa. Ciò spiega probabilmente perché la riflessione leopardiana sulla Spagna sia così spesso incentrata sul paragone tra l'antica situazione della terra iberica e la presente, destinato inevitabilmente a risolversi in una valutazione favorevole alla prima.

Ma procediamo innanzitutto nell'analisi dei passi incentrati sul confronto tra la Spagna e l'Italia, nei quali peraltro la questione della decadenza è ampiamente trattata. In un brano del 23 novembre 1823 Spagna e Italia sono

national d'Avignon, octobre 1990, dixième edition des journée d'études italiennes d'Avignon, Avignone, 1994, p. 73-83.

5. Rimando, per un approfondimento su questo tema, al saggio di Maria de las Nieves MuNIIZ MUNIIZ, "Sul concetto di decadenza storica in Leopardi», in Il pensiero storico e politico di Giacomo Leopardi, Atti del VI Convegno internazionale di studi leopardiani, Recanati, 9-11 settembre 1984, Firenze: Olschki, 1989, p. 285-306.

6. Si sofferma su tale questione Emilio GIORDANO nel saggio, «L'età delle macchine: appunti sul concetto leopardiano di "Storia" ", in Il pensiero storico e politico di Giacomo Leopardi, cit., p. 285-306. 
definite paesi meridionali dove «oggidì si vive poco [...] perché manca il corpo e il pascolo materiale e sociale delle illusioni, ma si pensa anche ben poco». «La Spagna», prosegue Giacomo, «'s̀ trovata finora nello stesso caso. Il suo clima, e la sua situazione geografica, e il governo ec. proteggevano le illusioni come in Italia, senza però lasciarle profittare, né proccurarsene punto di vita massime esterna e sociale». ${ }^{7}$ Incentrate invece sulle connessioni tra le vicende storico-politiche e l'espressione linguistico-letteraria di una nazione sono le carte 3855-3863, datate 10-11 novembre 1823:

Tra le cagioni del mancar noi (e così gli spagnuoli) di lingua e letteratura moderna propria, si dee porre, e per prima di tutte, la nullità politica e militare in cui è caduta l'Italia non men che la Spagna dal 600 in poi, epoca appunto da cui incomincia la decadenza ed estinzione delle lingue e letterature proprie in Italia e in Ispagna [...]. Come cagione assoluta, la nullità politica e militare degl'italiani e spagnuoli ha prodotto in mancar essi di lingua e letteratura moderna dal 600 in qua, ed il mancarne oggi. Essa nullità è cagione che l'Italia e la Spagna abbiano perduto d'allora in poi il loro essere di nazione. Quindi essa è cagione che l'Italia e la Spagna non abbiano, e d'allora in qua, né letteratura moderna, né filosofia ec. Esse non hanno lingua moderna propria, perché mancano di propria letteratura e filosofia moderna; ma di queste perché ne mancano? Perché non sono più nazioni; e nol sono, perché senza politica e senza milizia, non influiscono più sulla sorte degli altri, né sulla lor propria, non governano né si governano, e la loro esistenza o il loro modo di essere è indifferente al resto d'Europa [...]. Questa politica condizione dell'Italia e della Spagna ha prodotto e produce i soliti e immancabili effetti. Morte e privazione di letteratura, d'industria, di società, di arti, di genio, di coltura, di grandi ingegni, di facoltà inventiva, d'originalità, di passioni grandi, vive, utili o belle e splendide, d'ogni vantaggio sociale, di grandi fatti e quindi di grandi scritti, inazione, torpore così nella vita privata e rispetto al privato, come rispetto al pubblico, e come il pubblico è nullo rispetto alle altre nazioni [...]. Senz'attività, senza industria, senza spirito di letteratura, d'arti ec. senza spirito né uso di società, la vita degli spagnuoli e degl'italiani si riduce a una routine d'inazione, d'ozio, d'usanze vecchie e stabilite, di spettacoli e feste regolate dal Calendario, di abitudini ec. [...] E quel che dico dell'Italia, dico altresì della Spagna, la quale però, dal 600 in poi (come anche al suo buon tempo), vive e ha vissuto men dell'Italia, non p. altro se non perché meno communicando cogli stranieri, men vita ha ricevuto di fuori, non che p. se stessa ell'abbia avuto molto men vita di noi, e forse anche p. suo carattere è meno atta a tal comunione, e a ricevere la vita altrui. E quindi la sua lingua e letteratura, isterilendosi, decrescendo, scemando, perdendo e riducendosi a nulla quanto la nostra ha fatto, si è forse contuttociò meno imbarbarita ec. della nostra: che non so se si debba contare p. maggior male o bene ec. ${ }^{8}$

Il ragionamento espresso da Leopardi in queste pagine è ben chiaro: lo stato di decadenza in ambito politico e militare in cui si sono trovate l'Italia e

7. Giacomo LeOPaRDI, Zibaldone di Pensieri, cit., p. 278.

8. Ivi, p. 2029-2034. 
la Spagna dal diciassettesimo secoli in poi ha prodotto come diretta conseguenza un isterilimento della produzione letteraria e dello sviluppo linguistico dei due paesi. Non può insomma esistere, secondo Giacomo, una letteratura vitale in una nazione non vitale, così come non può esistere una lingua viva $\mathrm{e}$ aperta al rinnovamento in uno stato arretrato e impermeabile alle innovazioni. L'idea di uno stretto legame tra condizione socio-politica ed espressione letteraria di una nazione appare, anche alla luce del brano sopra citato, fortemente caratterizzante il pensiero del poeta per il quale, dunque, gli ambiti letterario e socio-politico si influenzano reciprocamente tanto da rendere impossibile la valutazione di una produzione letteraria prescindendo dall'analisi del contesto socio-politico in cui essa nasce. Molto interessante appare anche l'osservazione circa il maggior imbarbarimento della lingua e della letteratura italiana rispetto alla spagnola: ciò sarebbe da ricondurre ai frequenti contatti avuti dall'Italia con realtà straniere che invece sarebbero mancati alla Spagna. Quantunque Giacomo sospenda il giudizio, nell'ultima frase del passo citato, sull'eventuale negatività di tale stato di cose («che non so se si debba contare p. maggior male o bene»), sembra di poter dire che l'uso stesso del termine «imbarbarimento» sottintenda una valutazione globalmente negativa di tale frequente contatto con popoli stranieri.

Un carattere di notazione tecnica, e anzi quasi folkloristica, riveste invece l'ultimo dei passi dedicato al paragone tra Italia e Spagna. Si tratta di un brano della carta 4265, datata 30 marzo 1827, nel quale si afferma che il costume italiano di attribuire lunghi e pomposi titoli nobiliari, oggetto di derisione da parte di molti popoli europei, primi tra tutti i francesi, sarebbe stato mutuato dalle usanze spagnole. ${ }^{9}$

Ma tornando al tema socio-politico, così caro a Leopardi, ci si soffermerà innanzitutto su un passo datato 10 novembre 1820, il primo dello Zibaldone in cui il poeta tratta delle vicende iberiche:

Nella Spagna barbara di barbarie non primitiva ma corrotta per la superstizione, la decadenza da uno stato molto più florido, civile, colto e potente, gli avanzi de' costumi moreschi ec, nella Spagna, dico, l'ignoranza sosteneva la tirannia. Questa dunque doveva cadere ai primi lampi di una certa filosofia, derivati dall'invasione e dimora de' francesi, e dalla rivoluzione del mondo. L'ignoranza è come il gelo che assopisce i semi e gl'impedisce di germogliare, ma non gli uccide, come l'incivilimento, e passato l'inverno quei semi germogliano alla primavera. Così è accaduto nella Spagna, dove quel popolo, tornato quasi vergine ha sentito le scosse dell'entusiasmo, e l'avea già dimostrato nell'ultima guerra. E perciò s'è veduto quivi il contrario delle altre nazioni, come osserva l'autore del Manuscrit de S.te Hélène, ciò̀ che lo spirito rivoluzionario esisteva solamente in quelli che pel loro stato erano più colti, preti, frati, nobili, tutti quelli che nella rivoluzione non aveano che a perdere: perché il torpore della nazione non derivava da eccesso d'incivilimento, ma da difetto; e i pochi colti, probabilmente non lo erano all'eccesso, come altrove, ma

9. Ivi, p. 2383. 
quanto basta e conviene, e non più. Quando la Spagna sarà bene incivilita ricadrà sotto la tirannia, sostenuta non più dall'ignoranza, ma per lo contrario dall'eccesso del sapere, dalla freddezza della ragione, dall'egoismo filosofico, dalla mollezza, dal genio per le arti e gli studi pacifici. E questa tirannia sarà tanto più durevole e quanto più moderata della precedente. E se il re di Spagna avrà vera politica dovrà promuovere a tutto potere l'incivilimento del suo popolo (e in questi tempi vi potrà riuscire più facilmente e più presto). $\mathrm{E}$ con ciò non consoliderà la loro indipendenza come si crede comunemente ma gli assoggetterà di nuovo, e ricupererà quello che ha perduto. Non c'è altro stato intollerante di tirannia, o capace di esserne esente, fuorché lo stato naturale e primitivo, o una civilizzazione media, com'è ora quella della Spagna, com'era quella de' Romani ec. Atene e la Grecia quando furono sommamente civili, non furono mai libere veramente. ${ }^{10}$

In questo caso l'attenzione è focalizzata sulla tirannia, un regime di governo che avrebbe trovato in Spagna terreno fertile grazie all'ignoranza diffusa tra la popolazione. I più strenui oppositori della tirannia sono stati, in quel contesto, i ceti più colti della nazione (preti, frati, nobili). Ma quando gli spagnoli raggiungeranno un eccessivo grado di incivilimento, essi ricadranno sotto la tirannia, sostenuta questa volta non già dall'ignoranza ma «dalla freddezza della ragione, dall'egoismo filosofico, dalla mollezza, dal genio per le arti e gli studi pacifici». Dunque, un regime non democratico può instaurarsi, secondo Leopardi, in condizioni di "eccesso», sia di ignoranza che di cultura, in quanto i cittadini saranno in un caso privi degli strumenti intellettuali necessari per comprendere e contestare le azioni del proprio governo, in un altro troppo coinvolti dai propri interessi speculativi per promuovere forme di dissenso contro la tirannia. Le uniche situazioni nelle quali si prova intolleranza per la tirannia sono, per Giacomo, lo stato di media civilizzazione, proprio della Spagna a lui contemporanea, ma destinato in breve a mutarsi in un eccesso di civilizzazione, e soprattutto lo stato naturale e primitivo, ancora vagheggiato dal poeta, a questa altezza cronologica (si è già ricordato che tale passo è datato 10 novembre 1820 ), come una felice età aurorale e primigenia del mondo.

Dunque, l'immagine della Spagna che si delinea dalla lettura delle carte zibaldoniche finora citate è quella di una nazione simile all'Italia per la natura dei suoi abitanti, per le condizioni climatiche, per le usanze e i costumi, nonché per un diffuso stato di decadenza che l'ha privata tanto della primitiva vitalità quanto di una propria autonomia culturale e linguistica. Ma la Spagna è soprattutto, nell'ottica leopardiana, un paese scarsamente incivilito. Questa caratteristica, tuttavia, non necessariamente è del tutto negativa: la Spagna sembra infatti conservare, per Giacomo, le tracce di uno «spirito naturale», sorta di tenue ma resistente cordone ombelicale con quell' "età aurorale del mondo" che, nella fase del pessimismo storico, il poeta ancora poneva a monte del suo sistema speculativo. Su questo punto si consuma, a noi pare, un'insanabile divergenza con l'immagine leopardiana dell'Italia, paese invece 
troppo incivilito e dunque del tutto incapace di mantenere un seppur labile legame con la naturalità degli antichi, che la terra iberica ha invece conservato nonostante le spinte civilizzatrici della religione cristiana: "Osservate ancora la somma religione degli spagnuoli, la quale pur non bastava a storcere le loro inclinazioni naturali...». ${ }^{11}$

Completano l'immagine leopardiana della nazione spagnola due passi del febbraio 1821 di argomento «bellico». Il poeta intende qui delineare le virtù guerriere degli spagnoli, contestando innanzitutto l'opinione di Floro, scrittore latino di probabile origine iberica, che ebbe a definire gli spagnoli «promptius in pacis partes». Al contrario Giacomo, ricordando anche fatti storici di rilievo come il celebre assedio di Sagunto, sostiene che la nazione iberica, diventata interamente pacifica in tempi recenti, abbia però dato prova, nel passato, di eccellenti doti di combattente, nonché di uno spirito bellicosissimo e valorosissimo soprattutto nel difendersi dagli attacchi stranieri. ${ }^{12}$

Un discorso a parte meritano le valutazioni espresse da Leopardi sulla lingua spagnola. ${ }^{13}$ Si legga innanzitutto il passo datato 18 agosto 1822 :

La nazione spagnuola poetichissima per natura e per clima fra tutte l'Europee (non agguagliata in ciò che dall'Italia e dalla Grecia), e fornita di lingua poetichissima fra le lingue perfette (non inferiore in detta qualità se non all'italiana, e non agguagliata di gran lunga da nessun'altra) non ha mai prodotto un poeta e un poema che sia o sia stato di celebrità veramente europea. Tanto privilegiarono le istituzioni politiche alle qualità naturali. ${ }^{14}$

Giacomo riconosce dunque allo spagnolo un carattere poetico, anzi «poetichissimo» e lo classifica come idioma «perfetto» al pari dell'italiano. Egli attribuisce tuttavia alle istituzioni politiche iberiche la colpa di aver represso le qualità naturali di questa lingua e di averla resa così incapace di dar voce a un'opera letteraria di fama europea. Questo giudizio leopardiano appare singolare, e dunque in qualche misura criptico, vista l'acquisita notorietà di varie opere in spagnolo, tra le quali il celeberrimo Don Chisciotte di Miguel de Cervantes. $\grave{E}$ tuttavia significativo il fatto che nuovamente, e stavolta nell'ambito dell'espressione di un giudizio letterario, il poeta rilevi il contrasto tra stato naturale e civilizzazione, attribuendo a quest'ultima, incarnata dalle istituzioni politiche, caratteristiche di marcata negatività, così come riveste estrema importanza la riproposizione, stavolta in ambito linguistico-letterario, di quel paragone con l'Italia che tanta importanza riveste nell'analisi degli usi e costumi spagnoli. Del resto Leopardi coglie molteplici affinità tra l'italiano e lo spa-

11. Ivi, p. 996.

12. Cfr. ivi, p. 416-417.

13. Si veda, a questo proposito, il saggio di Diego Poli, «Lo spagnuolo, il "parallelo" e la lingua di Leopardi", in Rapporti culturali fra Italia e Spagna, VII Incontro, Macerata: Università degli Studi, 16-17 novembre 2000, supplemento a Quaderni di filologia e lingue romanze. Ricerche svolte nell'Università degli Studi di Macerata, terza serie, 15, 2000, p. 37-40.

14. Giacomo LeOPARDI, Zibaldone di Pensieri, cit., p. 1396. 
gnolo, finendo per impostare la riflessione zibaldonica sull'idioma iberico nella forma di un'insistita analogia con l'italiano. Le carte incentrate su tale questione risalgono al periodo tra il luglio e l'ottobre del 1823. Se il passo del 21 luglio 1823 si sofferma su un elemento tecnico, il frequente uso nell'italiano e nello spagnolo scritto del verbo «andare» nel senso di «essere», ${ }^{15}$ le carte 33903394 (10 settembre 1823) affrontano analiticamente la problematica della ricezione dei lemmi iberici nella lingua italiana:

La potenza avuta dagli Spagnuoli in Europa, e in Italia nominatamente, al tempo appunto che la lingua e letteratura nostra si formava e perfezionava, ciò fu nel cinquecento, fece che molte voci e molte più locuzioni e forme spagnuole fossero, non solo nel volgo e nel discorso familiare, ma dai dotti e dai letterati nella lingua scritta ed illustre italiana introdotte o accettate in quel secolo e nel seguente eziandio [...]. Le frasi e i modi, ed anche i vocaboli spagnuoli introdotti nella nostra lingua, stanno e conversano in essa colle nostre voci italiane così naturalmente che paiono non venuti ma nati, non ispagnuoli ma italiani quanto alcun altro mai possa essere e quanto lo sono i nostri propri vocaboli [...]. La lingua spagnuola è carnal sorella dell'italiana, non di famiglia solo e di nascita e di eredità, ma di volto, di persona e di costumi [...]. La lingua spagnuola è piuttosto altra che diversa dall'italiana. Ed era ben ragione che così fosse, perocché l'Italia, la Spagna e la Grecia sono in Europa per natura di clima, di terreno e di cielo le più conformi provincie meridionali. Or tra queste, la Spagna e l'Italia avendo l'una dato, l'altra ricevuto una stessissima lingua, era ben naturale che in processo di tempo ambedue riuscissero tanto e niente meno conformi di linguaggio, quanto a due separate nazioni è possibile il più. ${ }^{16}$

Il ragionamento si completa con quanto espresso nella carta 3730 , datata 18 ottobre 1823: il poeta osserva in questo caso che anche le voci e le frasi italiane entrate nell'idioma spagnolo a cavallo tra il Cinquecento e il Seicento sono a tutt'oggi di uso comune e non suonano affatto forestiere. ${ }^{17}$ Le affinità esistenti tra queste due lingue possono dunque, secondo Giacomo, essere definite di carnale sorellanza: ciò si deve al fatto che entrambe le nazioni derivano la loro lingua dal latino, ma anche a una stretta somiglianza di «natura, di clima, di terreno e di cielo»: ancora una volta in Leopardi il discorso linguistico-letterario appare inscindibile dalla riflessione di ambito socio-antropologico.

Qual è dunque, in conclusione, l'immagine della Spagna delineatasi nelle pagine dello Zibaldone? Gli elementi che con più forza emergono sono quelli di una nazione con un glorioso passato piombata in uno stato di decadenza da cui minimamente la riscatta una naturalità in parte preservata; una nazione pacifica ma altresì capace di guerreggiare, un paese di radicata tradizione religiosa, caldo e mediterraneo, in cui le vestigia dell'antichità si ergono a impe- 
ritura testimonianza di un glorioso passato. Ma soprattutto un paese profondamente affine all'Italia, rispetto alla quale si pone in un rapporto di stretta somiglianza antropologica, culturale, linguistica. E tuttavia la realtà spagnola appare, nella visione leopardiana, meno disastrata e disastrosa di quella italiana, forse per la visione più distaccata e dunque meno pessimistica che il poeta sviluppa verso una terra oggetto, sì, del suo ammirato interesse, ma pur sempre straniera, ma anche, probabilmente, per un barlume di «spirito naturale» che a Giacomo pare di individuare nella nazione spagnola e che tiene la terra iberica legata con un sottile filo d'acciaio a quell'aurorale età primigenia che la moderna Italia ha sepolto sotto le macerie della sua barbara civiltà. 